\title{
Development of Cytoplasmic Male Sterile IR24 and IR64 Using CW-CMS/Rf17 System
}

Kinya Toriyama* and Tomohiko Kazama

\begin{abstract}
Background: A wild-abortive-type (WA) cytoplasmic male sterility (CMS) has been almost exclusively used for breeding three-line hybrid rice. Many indica cultivars are known to carry restorer genes for WA-CMS lines and cannot be used as maintainer lines. Especially elite indica cultivars IR24 and IR64 are known to be restorer lines for WA-CMS lines, and are used as male parents for hybrid seed production. If we develop CMS IR24 and CMS IR64, the combination of $F_{1}$ pairs in hybrid rice breeding programs will be greatly broadened.

Findings: For production of CMS lines and restorer lines of IR24 and IR64, we employed Chinese wild rice (CW)type CMS/Restorer of fertility 17 (Rf17) system, in which fertility is restored by a single nuclear gene, Rf17. Successive backcrossing and marker-assisted selection of Rf17 succeeded to produce completely male sterile CMS lines and fully restored restorer lines of IR24 and IR64. CW-cytoplasm did not affect agronomic characteristics.
\end{abstract}

Conclusions: Since IR64 is one of the most popular mega-varieties and used for breeding of many modern varieties, the CW-CMS line of IR64 will be useful for hybrid rice breeding.

Keywords: Cytoplasmic male sterility, Fertility restorer, Hybrid rice

\section{Findings}

Hybrid rice has an average $15 \%$ to $20 \%$ yield advantage over inbred lines. Hybrid rice planted area accounts for $57.6 \%$ in China, $14.5 \%$ in the USA, $9.4 \%$ in Vietnam, $6.8 \%$ in Bangladesh, $4.3 \%$ in the Philippines, $3.2 \%$ in India, and $0.5 \%$ in Indonesia (Barclay 2010). Most commercial hybrid rice has been developed based on a three-line system, namely A (CMS line), B (maintainer line) and $\mathrm{R}$ (restorer line), involving the almost exclusive use of wild-abortive-type CMS (WA-CMS) lines, as it accounted for about $90 \%$ of the rice hybrids produced in China and $100 \%$ of the hybrids developed outside China (Sattari et al. 2007). Although two-line hybrids based on photoperiod- or thermo-sensitive genic male sterile lines are gradually increased in China up to onethird of the total hybrid rice growing area, WA-CMS still predominated (Huang et al. 2014). A major technical handicap in the development of hybrid rice using WA-CMS is a limited source of maintainer lines, as many indica elite cultivars are known to carry restorer genes for WA-CMS lines and cannot be used as

\footnotetext{
* Correspondence: torikin@bios.tohoku.ac.jp

Graduate School of Agricultural Science, Tohoku University, Sendai 981-8555, Japan
}

maintainer lines (Virmani 1994). For example, IR24 and IR64 are restorer lines for WA-CMS lines, and are used as male parents for hybrid seed production (Jing et al. 2001; Cai et al. 2013). Huge efforts had been made to establish maintainer lines for WA-CMS based on indica varieties through a maintainer-breeding program at the International Rice Research Institute (Virmani 1994). If we develop CMS-IR24 and CMS-IR64, the combination of $F_{1}$ pairs in hybrid rice breeding programs will be greatly broadened. For production of CMS lines and restorer lines with a nuclear background of elite indica cultivars, IR24 or IR64, we employed CW-CMS/Rf17 system, in which fertility is restored gametophytically by a single nuclear gene, $R f 17$ (Fujii and Toriyama 2009).

The Taichung 65 (T65) nuclear background CMS line, CWA with [cms-CW]rf17rf17, and the restorer line CWR with [cms-CW]Rf17Rf17 (Fujii and Toriyama 2009) were successively backcrossed with IR24 or IR64 by the methods of a biotron breeding system (Ohnishi et al. 2013). The presence or absence of $R f 17$ allele was detected by SNP in the promoter region at 2,286-bp upstream of the initiation codon; $R f 17$ carried $\mathrm{T}$, while $r f 17$ carried A (Fujii and Toriyama, 2009). This SNP was found within the recognition sequence (GTNAC) of 
Mae III. PCR-amplification of the promoter region using Primer F: TCGTTCACCACGGTAGATAGACTCAT and Primer R: CCCACATCTTCTCCTTGCATAATCC, followed by digestion with Mae III (PCR-RFLP analysis), yielded a $291 \mathrm{bp}$-band and a 79-bp band for the Rf17 allele, while yielding bands of $192 \mathrm{bp}, 99 \mathrm{bp}$ and $79 \mathrm{bp}$ for the $r f 17 \mathrm{al}$ lele (Fig. 1). During the backcrossing process, plants with rf17rf17 were completely sterile, while plants with Rf17rf17 set seeds (Fig. 2). Pollen grains were observed in the $\mathrm{BC}_{6} \mathrm{~F}_{1}$ plants of CWA $\mathrm{x}$ IR24 (rf17rf17), and in the $\mathrm{BC}_{4} \mathrm{~F}_{1}$ plants of CWR x IR24 (Rf17rf17). Pollen grains of CWA x IR24 were morphologically normal and filled with starch but did not germinate on stigma, while pollen grains of CWR $x$ IR24 germinated on stigma in the same manner as IR24 (Fig. 2). This is consistent with the previously observed pollen grains of CWA and CWR, which had the T65 nuclear background (Fujii and Toriyama 2005).

Agronomic characteristics were compared between IR24 and CMS-IR24 $\left(\mathrm{BC}_{6} \mathrm{~F}_{1}\right.$ plants of CWA $x$ IR24) for two years. All experimental materials were transplanted in the paddy field at a spacing of $20 \mathrm{~cm}$ between plants within rows and $30 \mathrm{~cm}$ between rows. No significant differences were found between the maintainer line and the

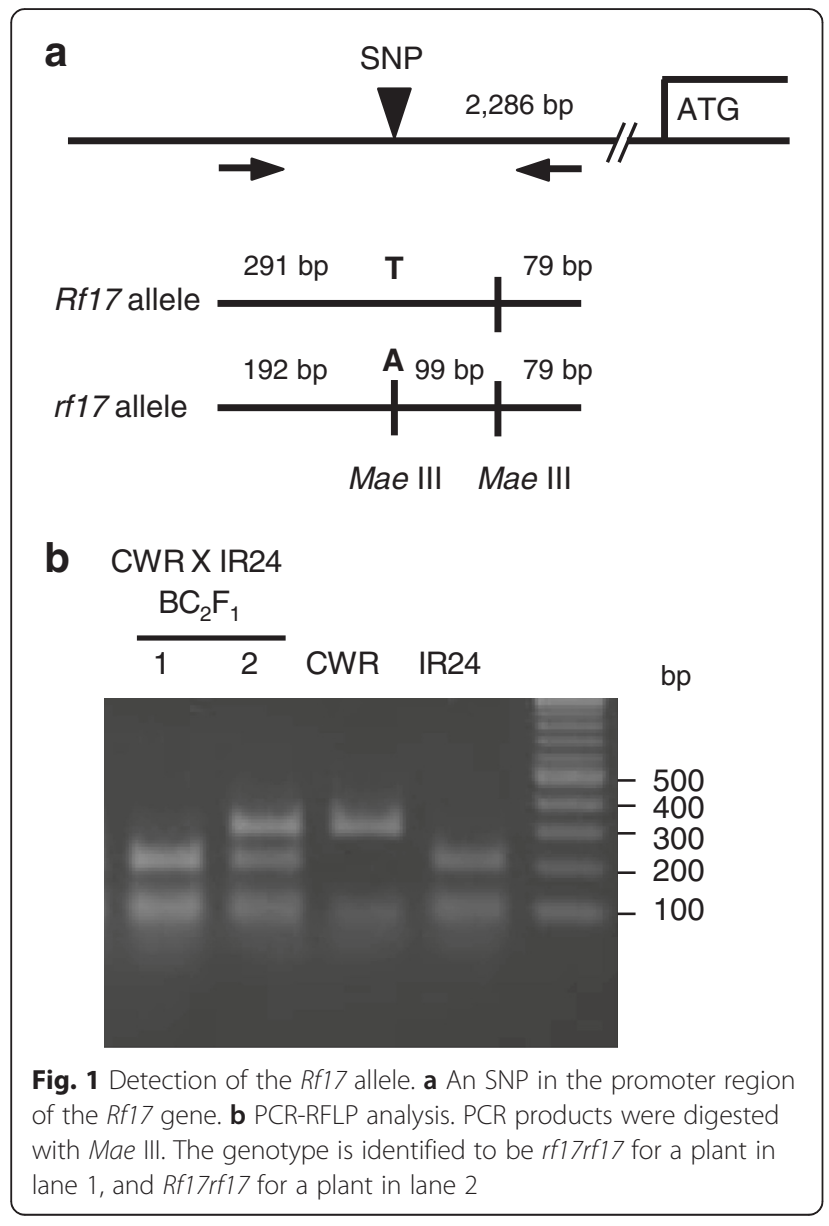

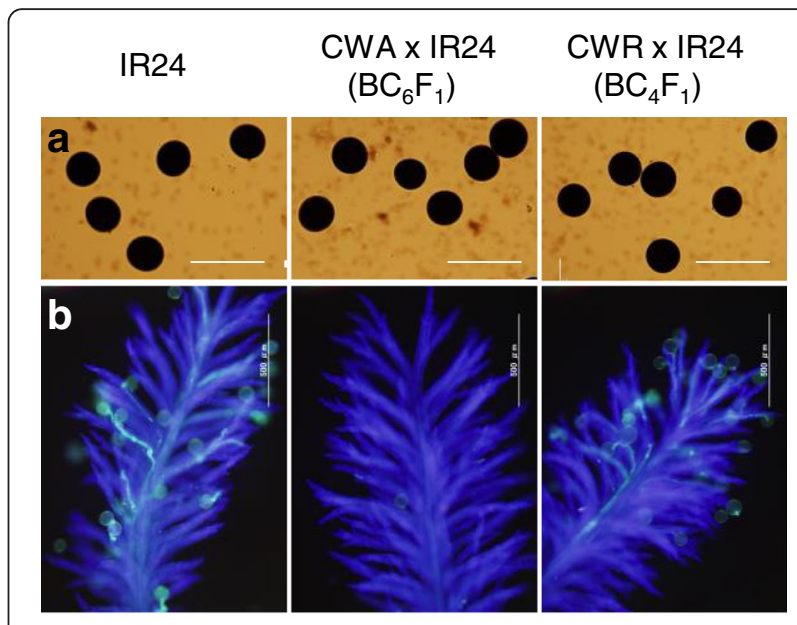

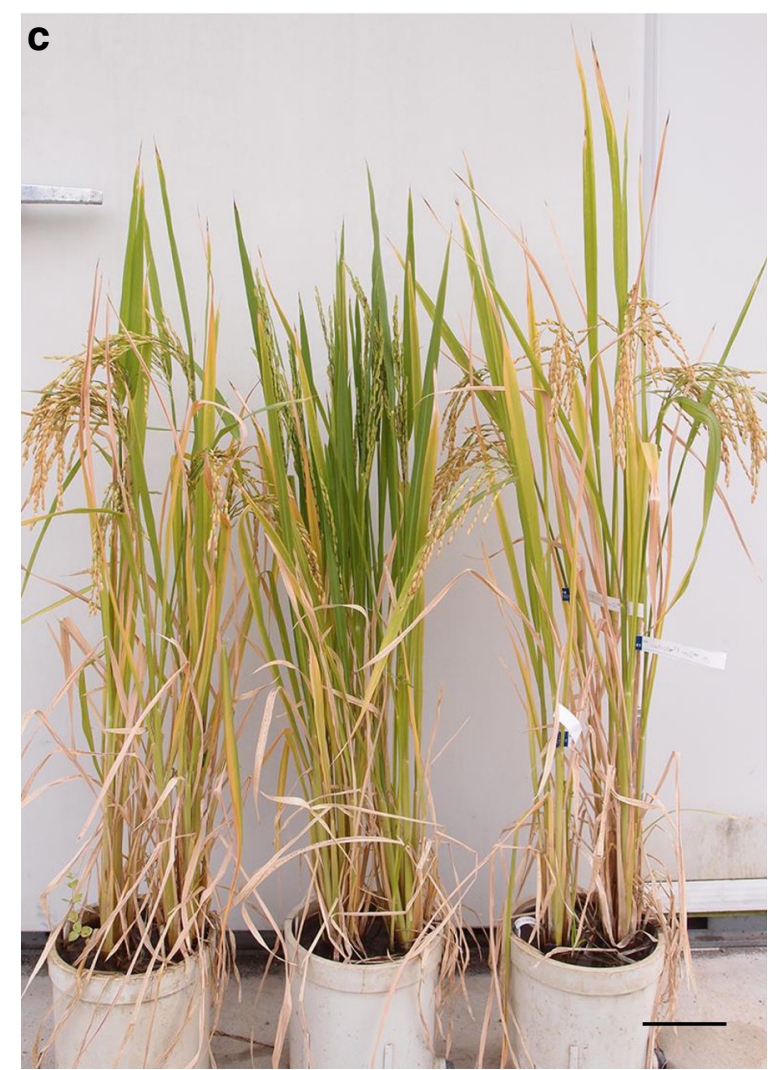

Fig. 2 Maintainer line (IR24), CMS line of IR24 (CWA x IR24 BC ${ }_{6} F_{1}$ ) and restorer line of IR24 (CWR $\times$ IR24, $\left.\mathrm{BC}_{4} \mathrm{~F}_{1}\right)$. a Pollen grains stained with $\mathrm{I}_{2}-\mathrm{Kl}$. b Pollen tubes on stigma stained with aniline blue. $\mathbf{c}$ Plants grown in a pot. Bar $=200 \mu \mathrm{m}(\mathbf{a}), 500 \mu \mathrm{m}(\mathbf{b})$ and $10 \mathrm{~cm}$ (c)

CMS line in stem length, panicle length, length of exserted panicle, distance between panicle base and auricle of flag leaf, number of panicle per plant, heading date and anthesis time (Table 1, Additional file 1: Table S1). Comparison was also done for IR64 and $\mathrm{BC}_{4} \mathrm{~F}_{1}$ or $\mathrm{BC}_{6} \mathrm{~F}_{1}$ plants of CWA $\mathrm{x}$ IR64. No deleterious characteristics were observed in the CMS-IR64 line, although panicles of the CMS line were slightly 
Table 1 Comparison of agronomic characteristics between CMS line and maintainer line in 2014

\begin{tabular}{|c|c|c|c|c|}
\hline \multirow{3}{*}{$\begin{array}{l}\text { Year } \\
\text { Lines }\end{array}$} & \multicolumn{4}{|l|}{$2014(n=4)$} \\
\hline & $\begin{array}{l}\text { CWA } \times \text { IR24 } \\
\left(\mathrm{BC}_{6} \mathrm{~F}_{1}\right)\end{array}$ & IR24 & $\begin{array}{l}\mathrm{CWA} \times \mathrm{IR64} \\
\left(\mathrm{BC}_{4} \mathrm{~F}_{1}\right)\end{array}$ & IR64 \\
\hline & CMS & Maintainer & CMS & Maintainer \\
\hline Stem length $(\mathrm{cm})$ & $49.9 \pm 0.8$ & $50.8 \pm 0.8$ & $65.7 \pm 1.6$ & $67.4 \pm 0.7$ \\
\hline Panicle length (cm) & $22.6 \pm 0.6$ & $22.7 \pm 0.3$ & $29.4 \pm 0.3$ & $28.9 \pm 0.2$ \\
\hline Length of exserted panicle $(\mathrm{cm})$ & $22.1 \pm 0.7$ & $22.6 \pm 0.3$ & $29.0 \pm 0.1$ & $28.9 \pm 0.2$ \\
\hline $\begin{array}{l}\text { Distance between panicle base } \\
\text { and auricle of flag leaf }(\mathrm{cm})\end{array}$ & $-0.5 \pm 0.4$ & $0.4 \pm 0.3$ & $1.4 \pm 1.0$ & $2.9 \pm 1.0$ \\
\hline Panicle exsertion rate (\%) & 98 & 100 & 100 & 100 \\
\hline Number of panicle per plant & $11.0 \pm 0.8$ & $13.8 \pm 0.9$ & $19.3 \pm 2.3$ & $17.8 \pm 0.7$ \\
\hline Heading date & Aug. 14 & Aug. 14 & Aug. 14 & Aug. 14 \\
\hline Anthesis time & 8:00-11:00 & 8:00-11:00 & $8: 30-10: 30$ & $8: 30-10: 30$ \\
\hline
\end{tabular}

larger than those of the maintainer line in the year of 2015 (Table 1, Additional file 1: Table S1).

In order to enhance outcrossing, we shook the male parents with a stick to disperse pollen grains daily at peak anthesis for a period of one week at the time of flowering in 2014. The outcrossing rate was $55.5 \pm 3.9 \%$ for the CMS-IR24 and $23.9 \pm 1.4 \%$ for the CMS-IR64, while the seed setting rate of the maintainer line was $74.3 \pm 2.5 \%$ for IR24 and $72.8 \pm$ $2.2 \%$ for IR64 in 2014, and $65.2 \pm 1.0 \%$ for IR24 and $79.0 \pm 2.7 \%$ for IR64 in 2015 .

The seed setting rate of the restorer lines was 82.8 $\pm 3.9 \%$ for $\mathrm{BC}_{4} \mathrm{~F}_{1}$ plants of CWR x IR24 (Rf17rf17) when tested in 2014 (Fig. 2). It was $73.5 \pm 3.1 \%$ for $\mathrm{BC}_{5} \mathrm{~F}_{2}$ plants of CWR $\mathrm{x}$ IR24 (Rf17Rf17), and 84.4 \pm $2.7 \%$ for $\mathrm{BC}_{5} \mathrm{~F}_{2}$ plants of CWR x IR64 (Rf17Rf17) in 2015. These results demonstrated that the fertility of the restorer line was recovered up to that of the maintainer line.

In conclusion, we produced CMS lines of IR24 or IR64 nuclear background, which did not set any seeds. The fertility is fully recovered by the presence of $R f 17$. CW cytoplasm did not affect agronomic characteristics. The $R f 17$ allele was not detected in 69 accessions of the NIAS world rice core collection (Kojima et al. 2005) provided by the National Institute of Agrobiological Sciences Genebank (Tsukuba, Japan) or in any CMS lines so far tested, except for our CWR line (Toriyama et al. 2009). We expect that the CW-CMS/Rf17 system will be used for production of CMS lines of many indica cultivars. Efforts are now in progress to test if the CW-cytoplasm confers CMS in other elite indica cultivars. IR64 is one of the most popular mega-varieties and used for breeding of many modern varieties in South-east Asia (Septiningsih et al. 2009). The CW-CMS line of IR64 will be useful for hybrid rice breeding.

\section{Additional file}

Additional file 1: Table S1. Comparison of agronomic characteristics between CMS line and maintainer line in 2015. (XLS 35 kb)

\section{Abbreviations}

CMS: cytoplasmic male sterility; CW-CMS: Chinese wild rice-type CMS; Rf: restorer of fertility; T65: Taichung 65; WA-CMS: wild-abortive-type CMS.

\section{Competing interests}

The authors declare that they have no competing interests.

Authors' contributions

KT and TK conceived, designed, performed the experiments and wrote the manuscript. Both authors read and approved the final manuscript.

\section{Acknowledgments}

This work was supported by Science and technology research promotion program for agriculture, forestry, fisheries and food industry (Nos. 26010A; 26010AB).

Received: 18 November 2015 Accepted: 4 May 2016

Published online: 11 May 2016

\section{References}

Barclay A (2010) Hybridizing the world. Rice Today 9:32-35

Cai J, Liao QP, Dai ZJ, Zhu HT, Zeng RZ, Zhang ZM, Zhang GQ (2013) Allelic differentiation and effects of the Rf3 and Rf4 genes on fertility restoration in rice with wild abortive cytoplasmic male sterility. Biol Plantarum 57:274-280. doi:10.1007/s10535-012-0294-9

Fujii S, Toriyama K (2005) Molecular mapping of the fertility restorer gene for msCW-type cytoplasmic male sterility of rice. Theor Appl Genet 111:696-701. doi:10.1007/s00122-005-2054-0

Fujii S, Toriyama K (2009) Suppressed expression of RETROGRADE-REGULATED MALE STERILITY restores pollen fertility in cytoplasmic male sterile rice plants. Proc Natl Acad Sci U S A 106:9513-9518. doi:10.1073/pnas.0901860106

Huang JZ, ZG E, Zhang HL, Shu QY (2014) Workable male sterility systems for hybrid rice: Genetics, biochemistry, molecular biology, and utilization. Rice 7: 13. doi:10.1186/s12284-014-0013-6

Jing RC, Li XM, Yi P, Zhu YG (2001) Mapping fertility-restoring genes of rice WA cytoplasmic male sterility using SSLP markers. Bot Bull Acad Sinica 42:167-171

Kojima Y, Ebana K, Fukuoka S, Nagamine T, Kawase M (2005) Development of an RFLP-based rice diversity research set of germplasm. Breed Sci 55:431-440

Ohnishi T, Yoshino M, Toriyama K, Kinoshita T (2013) Rapid establishment of introgression lines using cytoplasmic male sterility and a restorer gene in 
Oryza sativa cv. Nipponbare. Mol Breeding 32:831-839. doi:10.1007/s11032013-9910-4

Sattari M, Kathiresan A, Gregorio GB, Hernandez JE, Nas TM, Virmani SS (2007) Development and use of a two-gene marker-aided selection system for fertility restorer genes in rice. Euphytica 153:35-42. doi:10. 1007/s10681-006-9213-5

Septiningsih EM, Pamplona AM, Sanchez DL, Neeraja CN, Vergara GV, Heuer S, Ismail AM, Mackill DJ (2009) Development of submergence-tolerant rice cultivars: the Sub1 locus and beyond. Ann Bot 103:151-160. doi:10. 1093/aob/men206

Toriyama K, Fujii S, Toda T, Itabashi E, Yamada M, Kazama T (2009) Molecular analysis of CW-type cytoplasmic male sterility and Rf17-mediated fertility restoration for hybrid rice breeding. SABRAO J Breed Genet 41:1-13, Special Supplement August 2009 CD

Virmani SS (1994) Heterosis and Hybrid Rice Breeding. Springer, Berlin, Heidelberg

\section{Submit your manuscript to a SpringerOpen ${ }^{\circ}$ journal and benefit from:}

- Convenient online submission

- Rigorous peer review

- Immediate publication on acceptance

- Open access: articles freely available online

- High visibility within the field

- Retaining the copyright to your article

Submit your next manuscript at $>$ springeropen.com 